\title{
Combustibles, comportamiento del fuego y emisiones en un pastizal y una sabana artificiales en Chiapas
}

\author{
Dante Arturo Rodríguez Trejo ${ }^{1 *}$, Pedro Martínez Muñoz ${ }^{2}$, Jorge Alberto Pulido Luna ${ }^{3}$, \\ Pedro Jerónimo Martínez Lara ${ }^{2}$ \& José Domingo Cruz López ${ }^{4}$ \\ 1. División de Ciencias Forestales, Universidad Autónoma Chapingo, Edo. de Méx., México; dantearturo@yahoo.com \\ 2. Biomasa, A. C., Villaflores, Chiapas, México; pmtz29@hotmail.com; pmtz_19@hotmail.com \\ 3. Escuela Técnica Superior de Ingeniería Agraria, Universidad de Lerida, Lerida, España; jorgepulidoluna@gmail.com \\ 4. Reserva de la Biosfera La Sepultura, Comisión Nacional de Áreas Naturales Protegidas, Jiquipilas, Chiapas, México; \\ pescadito130@hotmail.com \\ * Correspondencia
}

Recibido 25-II-2020. Corregido 12-III-2020. Aceptado 24-III-2020.

\begin{abstract}
Forest fuels, fire behavior and emissions in artificial grasslands and savannas in Chiapas, Mexico. Introduction: Burns are part of the management of introduced grasses in Chiapas, Mexico, and this may derive in forest fires. Objectives: To determine fuel load, fire behavior, $\mathrm{CO}_{2}$ emissions, and to get firefighting security issues for jaragua (Hyparrhenia rufa Nees.) grasslands and savannas. Methods: An artificial jaragua grassland and an artificial jaragua savanna were studied at the California and Flores Magón communities, respectively, in La Sepultura Biosphere Reserve. Were measured pre and post-fire fuel loads. Six prescribed burns (three heading and three backing fires) were conducted in each, grassland and savanna, and were measured meteorological as well as fire behavior variables. Emissions were estimated multiplying the consumed fuel load by an emission constant. Results: In the grassland, were obtained the following averages: fuel load, $6.214 \mathrm{t} /$ ha; residual load, 0.107 t/ha, and $\mathrm{CO}_{2}$ emission, $10.449 \mathrm{t} \mathrm{CO}_{2}$ /ha. For the savanna, were recorded 14.119, 2.161 and $20.460 \mathrm{t} \mathrm{CO}_{2} /$ ha, respectively, without differences for the pre and post-fire fuel loads between heading and backing fires. For the grassland, the heading fires reached $3.92 \mathrm{~m}, 1.83 \mathrm{~m}$ and $22.3 \mathrm{~m} / \mathrm{min}$ for flame length, flame depth and fire propagation rate, while for backing fires such values were, respectively: 1.07, 0.23 and $0.67 \mathrm{~m} / \mathrm{min}$, with significant differences. For the savanna, heading fires yielded $5.89 \mathrm{~m}$ (flame length), $1.53 \mathrm{~m}$ (flame depth) and $45.5 \mathrm{~m} / \mathrm{min}$ (propagation rate), while for backing fires that values were $2.21,0.76$ and $2.8 \mathrm{~m} /$ min, also with significant differences. Conclusions: Under the studied environmental conditions, particularly in the savanna, fire behavior is dangerous so a good prescription and more care must be taken for conducting controlled or prescribed burns. For forest fires, direct firefighting by the head of the fire must be avoided, for is too dangerous; instead it is recommended a firefighting by the back of the fire as well as waiting for a backing fire-advance of it after the fire reaches a ridge.
\end{abstract}

Key words: Chiapas, $\mathrm{CO}_{2}$, Hyparrhenia rufa, Jaragua grass, fire behavior, fuel consumption, fuel load.

Rodríguez Trejo, D.A., Martínez Muñoz, P., Pulido Luna, J.A., Martínez Lara, P.J., \& Cruz López, J.D. (2020). Combustibles, comportamiento del fuego y emisiones en un pastizal y una sabana artificiales en Chiapas. Revista de Biología Tropical, 68(2), 641-654.

En la depresión central de Chiapas, México, uno de los principales tipos de vegetación es la selva baja caducifolia (Rocha, Ramírez, \& González, 2010; Miranda, 2016); sin embargo, en esta zona se introdujeron pastos exóticos que se hicieron invasores.
Las especies invasoras compiten con las nativas, alteran el hábitat y las redes tróficas, contribuyen a la extinción de especies, entre otros impactos. Hasta 2007 en México se habían registrado 665 especies de este tipo (Aguirre et al., 2009). Una de ellas es el pasto 
jaragua, Hyparrhenia rufa Nees, ampliamente distribuido en la depresión central del estado, se cultiva en la parte baja de las montañas y de ahí va ganando terreno en claros, pues la alteración facilita su invasión.

En algunas áreas forma pastizales artificiales y, en otras, por la presencia de árboles, conforma sabanas artificiales. Este pasto generalmente es perenne, con tallos de hasta $2.5 \mathrm{~m}$ de altura. Fue introducido accidentalmente de África hasta América por barcos de esclavos y después se naturalizó, aunque algunos botánicos sitúan el este de América tropical como parte de su área de distribución natural. La especie es cultivada en México, Cuba, Nicaragua, Puerto Rico, Costa Rica, Brasil, Venezuela, Colombia y Brasil, para alimentar ganado (Bogdan, 1997; Lesur, 2010).

En el estado, el país y otras naciones, el jaragua es renovado con el uso del fuego; debido a las importantes superficies que cubre, así como su continua expansión tanto en el municipio como en la Reserva de la Biosfera La Sepultura (REBISE), al uso del fuego para su renovación, así como a los incendios originados por colillas y cerillos junto a vías de comunicación, en estos pastizales se producen incendios forestales.

También es conocido que el fuego mejora productividad y calidad de los pastos para el forraje, aunque en el estado de Nayarit, Villanueva, Negrete, Villalobos y Britton (2008) encontraron que el fuego no mejoró el rendimiento de $H$. rufa, pero sí su calidad forrajera.

En sitios sucesionales jóvenes de bosque tropical caducifolio, en el Parque Guanacaste, Costa Rica, las quemas anuales de H. rufa y otras poáceas pueden ser la causa de los aumentos en los contenidos de nutrimentos en el suelo, en especial Ca, Mg y K (Certini, 2005; Leiva, Rocha, Mata, \& Gutiérez, 2009).

Las sabanas naturales en la región de estudio son dominadas por árboles como hoja man (Curatella americana L.), nanche (Byrsonima crassifolia (L.) H.B.K.), quebracho (Acacia milleriana Standl), encinos (Quercus spp.) y otras. Las sabanas se originan en sitios con drenaje insuficiente donde pocas especies arbóreas toleran la saturación de humedad en el suelo, o bien por fuego antropógena. Este tipo de vegetación se deriva de selvas altas, selvas bajas, encinares y pinares de zonas secas (Miranda, 2016). Entre los géneros de los pastos componentes de las sabanas, los principales son: Andropogon, Aristida, Paspalum, Trichachne e Imperata (Miranda \& Hernández, 2014). En el área de estudio, prevalecen pastizales y sabanas con pastos exóticos.

El estudio de cargas de combustibles forestales es relevante para: determinar áreas de peligro de incendio forestal, manejo de combustibles forestales, estimar el comportamiento del fuego, emisiones de gases de invernadero y contaminantes, entre otros objetivos. Las cargas elevadas confieren más intensidad al fuego, pero son los combustibles ligeros, como los pastos y la hojarasca, los que le dan más velocidad de propagación al fuego (Scott, Bowman, Bond, Pyne, \& Alexander, 2014). El estudio del comportamiento del fuego, proporciona información para: la construcción de índices de peligro forestal, el control de incendios forestales, la realización de quemas prescritas y quemas controladas, una mayor seguridad para el combate de incendios forestales, la capacitación de combatientes de incendios forestales y la de ejecutores de quemas prescritas, y la capacitación de comunidades rurales que realizan quemas controladas.

El cambio climático global genera alteraciones como más incendios, que son más: extensos, intensos, severos, difíciles, peligrosos y caros de controlar (Scott et al., 2014). La quema de bosques tropicales favorece el establecimiento de sabanas, con pastos inflamables, aumenta la frecuencia de los incendios y contribuye al cambio climático global (Beerling \& Osborne, 2006).

En el municipio de Villaflores y la REBI$\mathrm{SE}$, algunas organizaciones e instituciones de investigación y enseñanza, realizan esfuerzos hacia un manejo integral del fuego, buscando reducir incendios forestales no deseados, en parte con un uso limitado y juicioso del fuego tanto como factor ecológico en ecosistemas donde esto es posible, como en el manejo 
tradicional de la tierra, por productores. Este estudio es parte de tales esfuerzos.

Este trabajo tiene como objetivos estimar y comparar las cargas de combustibles forestales, su consumo, el comportamiento del fuego y las emisiones de $\mathrm{CO}_{2}$ en quemas prescritas, a favor de viento y pendiente y en su contra, en un pastizal y en una sabana artificiales de pasto jaragua.

\section{MATERIALES Y MÉTODOS}

Áreas de estudio: Se seleccionaron dos: un pastizal artificial, con pasto jaragua $<1 \mathrm{~m}$ de altura y una sabana artificial, con pasto jaragua de $2 \mathrm{~m}$ de altura. La primera se ubicó en la comunidad California, en un terreno de 2 ha vecino al campamento de la REBISE, Villaflores, Chiapas, mientras que el segundo se enclavó en 1 ha del paraje "el jobo", en la comunidad Flores Magón, en la misma Reserva.

Para el pastizal, sobre una ladera se establecieron seis parcelas rectangulares de $20 \mathrm{x}$ $30 \mathrm{~m}$. La pendiente de las laderas alcanza hasta $70 \%$ y tiene una exposición dominante S. La vegetación original fue bosque de pino-encino (Pinus oocarpa Schiede y Quercus spp.), pero hace años se cambió el uso del suelo y se sembró el pasto jaragua.

Para la sabana, sobre las laderas que confluyen en un parteaguas fueron establecidas seis parcelas rectangulares de $20 \times 40 \mathrm{~m}$. Tres parcelas contiguas fueron colocadas a un lado del "parteaguas" (línea divisoria) y las otras tres, también contiguas, del otro lado. Las pendientes varían entre $21-70 \%$, una ladera del parteaguas tiene exposición NE y la otra exposición SO. La vegetación original fue selva baja caducifolia, pero hace años se deforestó y sembró pasto jaragua, constituyendo esta sabana artificial. Los principales árboles presentes son encinos (Quercus spp.) y nanches (Byrsonima crassifolia).

Quemas prescritas: En el pastizal, la mitad de las parcelas fueron quemadas a favor de pendiente y viento (Qf) y la otra mitad en contra (Qc), en marzo 2018. El tratamiento de cada parcela se asignó al azar. Para realizar las quemas primero se formuló un plan de quema prescrita y una prescripción, conforme a la Norma Oficial Mexicana de Uso del Fuego (NOM-015, Semarnat/Sagarpa 2009) y se notificó y recibió autorización de la Delegación Federal de la Secretaría de Medio Ambiente y Recursos Naturales (Semarnat) y de la Gerencia Estatal de la Comisión Nacional Forestal (Conafor) para hacerlas, con el propósito principal de realizar la investigación. Otros propósitos de la quema prescrita, no de investigación, fueron la generación de forraje tierno para el ganado y la reducción de combustibles forestales para abatir peligro de incendio forestal, así como la capacitación. Se siguió el protocolo del Sistema para el Manejo de Incidentes, para dar la mayor seguridad durante las quemas.

Los autores dirigieron y participaron en la ejecución y medición de las quemas, así como dos brigadas comunitarias (con elementos de los ejidos California y Flores Magón, principalmente), una para la realización de las quemas prescritas (16 elementos) y otra de apoyo para la medición (seis elementos). En el pastizal, el lado sur del terreno estaba delimitado por un camino de terracería, pero en el perímetro de los otros tres lados se abrió brecha cortafuego de $3 \mathrm{~m}$ de ancho. Asimismo, cada una de las seis parcelas de quema fue rodeada por una brecha cortafuegos de $2 \mathrm{~m}$ de ancho. En las Qc, se inició la quema por el lado más alto de la parcela y a 1-2 $\mathrm{m}$ de distancia se quemó otra línea paralela a la anterior, para que las llamas de la primera línea se tendieran hacia abajo de la ladera, reduciendo aún más la posibilidad de escape. Si se consideraba necesario se repetía este procedimiento con una faja más baja. Después se dejó que el fuego corriera hacia abajo, adelantándolo 1 o $2 \mathrm{~m}$ por los flancos para lograr un mayor control. Para las parcelas con quema a favor, se quemó de la forma descrita a los primeros metros de la parte alta de la parcela y luego se hizo ignición en el lado de la base, de modo que el fuego avanzara a favor (Fig. 1A). Para la sabana se quemó primero la parcela uno en contra (de la forma ya descrita en pastizal), luego la cuatro a favor; después 
A

Parteaguas

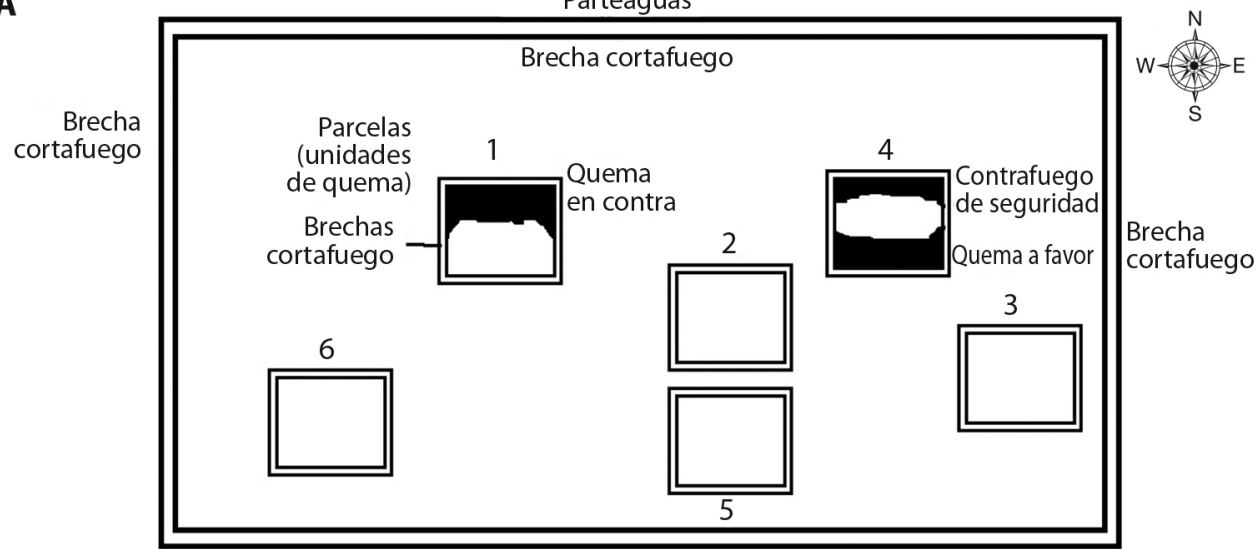

Camino de terracería

B

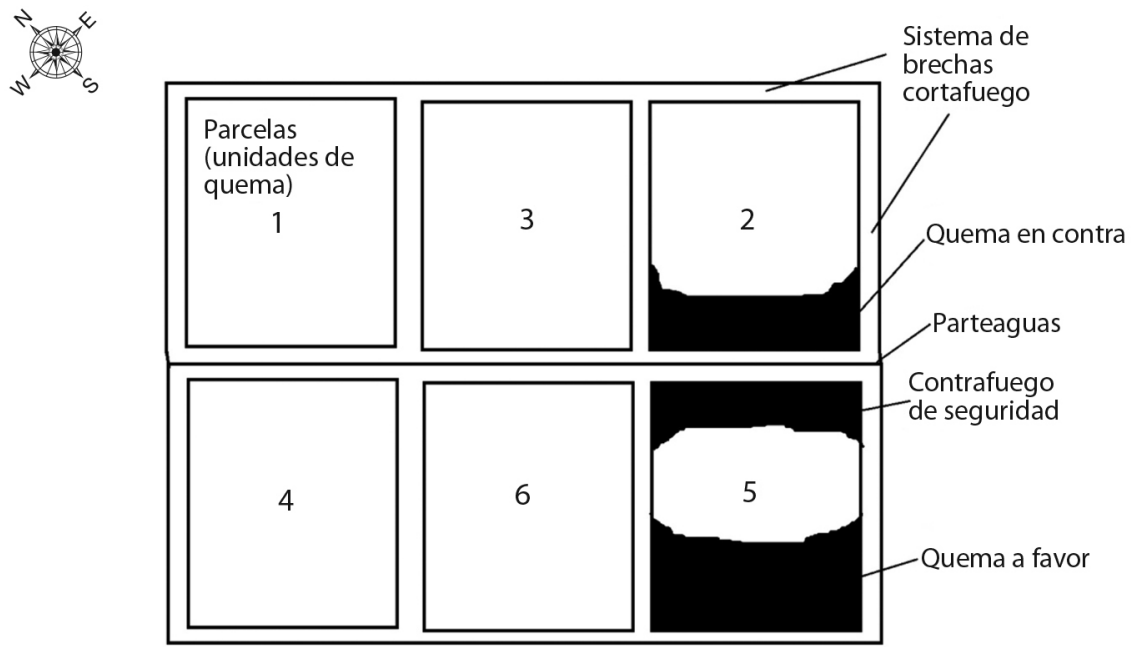

Fig. 1. Diseño experimental en A) pastizal y B) sabana.

Fig. 1. Experimental design for $\mathbf{A}$ ) grassland and $\mathbf{B}$ ) savanna.

la dos en contra y la cinco a favor. Finalmente se quemó la parcela seis a favor y la tres en contra (Fig. 1B).

\section{Muestreo de combustibles forestales:} Para el muestreo de combustibles forestales, se usaron unidades muestrales con forma de triángulo equilátero y $12 \mathrm{~m}$ por lado. La base del triángulo se enclavó paralela a la curva de nivel. Las líneas del triángulo fueron divididas en segmentos de 2, 4 y $12 \mathrm{~m}$, para el conteo de intersecciones con los materiales leñosos de $1 \mathrm{~h}$ de tiempo de retardo (TR) $(<0.6 \mathrm{~cm}$ de diámetro), $10 \mathrm{~h}$ TR $(0.6-2.5 \mathrm{~cm})$ (ambas en el segmento de $2 \mathrm{~m}), 100 \mathrm{~h}$ TR (2.5-7.5 $\mathrm{cm})$ (segmento de $4 \mathrm{~m}$ ) y $1000 \mathrm{~h}$ TR $(>7.5$ $\mathrm{cm})$ (segmento de $12 \mathrm{~m}$ ) (Brown, 1974), estos últimos tanto firmes como podridos. Al inicio de cada línea del triángulo de muestreo se ubicó un cuadro de $0.3 \times 0.3 \mathrm{~m}$ para hojarasca y capa de fermentación, de los que se estimó cobertura (\%) visualmente y se les midió cuatro profundidades (una en la parte media de cada lado) con regla. También al inicio de cada línea se enclavó un cuadro de 1 x $1 \mathrm{~m}$, donde se registró altura (4 mediciones) y cobertura 
(estimación visual, \%) de: zacates, herbáceas dicotiledóneas, arbustos y regeneración. En el lado de la base del triángulo, a $2 \mathrm{~m}$ del inicio, se levantaron nuevamente los datos en cuadros de $0.3 \times 0.3 \mathrm{~m}$ y el de $1 \times 1 \mathrm{~m}$, pero aquí se cosechó todo el material, colocado en bolsas de papel estraza y de polietileno, además de ser etiquetadas. Este material se trasladó a para su secado en estufas al Laboratorio de Zootecnia de la Universidad Autónoma de Chiapas, campus Villaflores. El material fue secado a $70{ }^{\circ} \mathrm{C}$ hasta obtener peso constante (peso seco). Con la medición de los combustibles en campo y la obtención de su peso seco, se calculó el peso seco por unidad de volumen medido en campo, que sirvió para la estimación del peso seco de los materiales a partir de sus medidas de campo. Después de la realización de la quema prescrita en cada parcela, el triángulo de muestreo fue remuestreado para registrar los combustibles residuales al fuego (Fig. 2). La metodología empleada para estimar los materiales leñosos se fundamentó en Brown (1974).

Muestreo de comportamiento del fuego y tiempo atmosférico: Perpendicular a la línea base del triángulo de muestreo, cruzando por su mitad, se colocó una línea de $15 \mathrm{~m}$ de longitud. En su inicio y a cada $5 \mathrm{~m}$ de distancia, se colocaron estadales de $1 \mathrm{~m}$ (para pastizal) y de $2 \mathrm{~m}$ de longitud (para sabana), marcados cada $20 \mathrm{~cm}$ (rojo y blanco alternados), que sirvieron para medir la longitud de llama (Lll). En su base, también perpendicular a las curvas de nivel, se colocó una regla de madera sobre el piso, con $1 \mathrm{~m}$ de longitud, también marcada con rojo y blanco en tramos de $20 \mathrm{~cm}$. Ésta se usó para medir profundidad de llama (Pll). $\mathrm{La}$ velocidad de propagación del fuego $(\mathrm{Vp})$ se obtuvo a partir del tiempo que el fuego tardó en desplazarse entre estadales. Lll y Pll se midieron cuando el fuego alcanzaba cada estadal (Fig. 3).

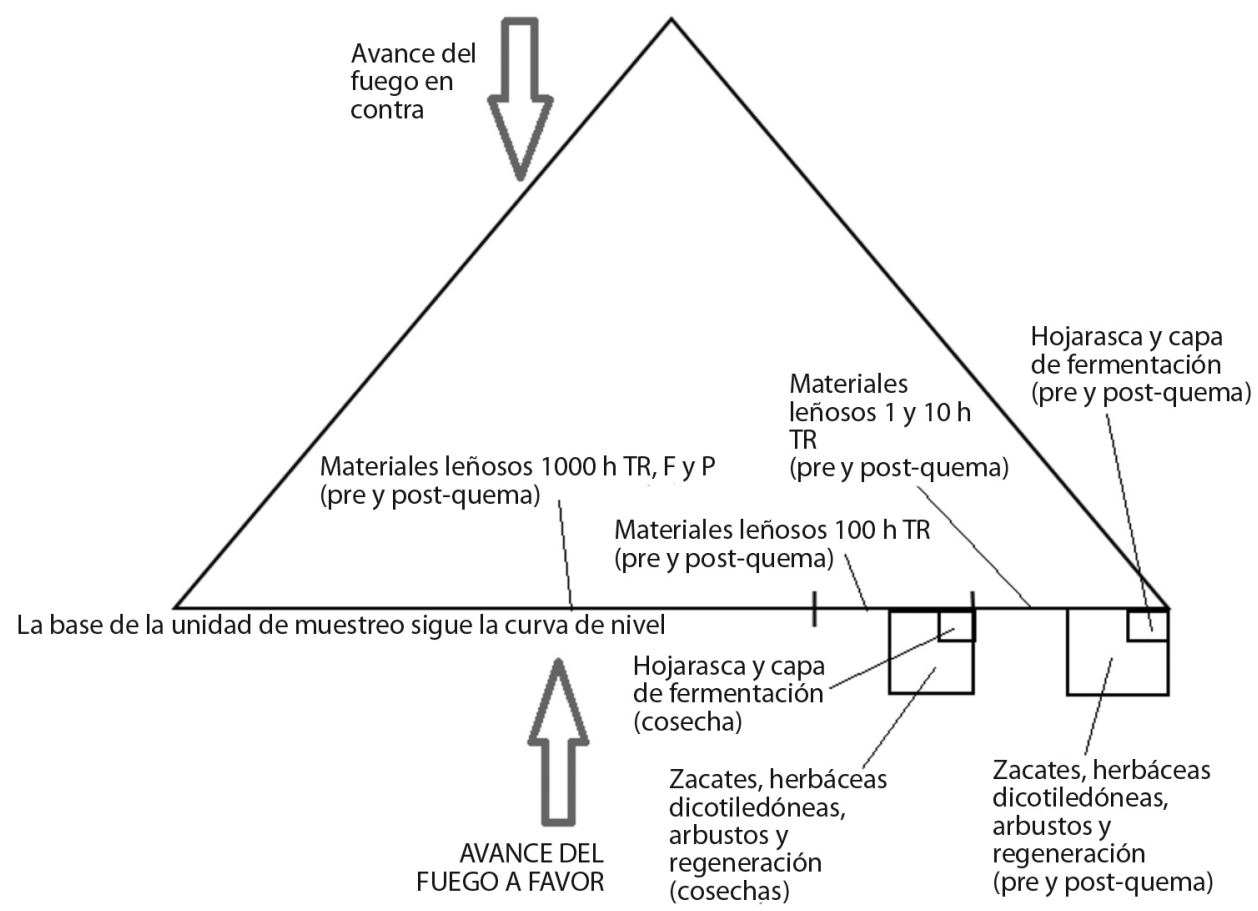

Fig. 2. Unidad de muestreo para los combustibles forestales en el pastizal y la sabana.

Fig. 2. Sampling unit for forest fuels in grassland and savanna. 


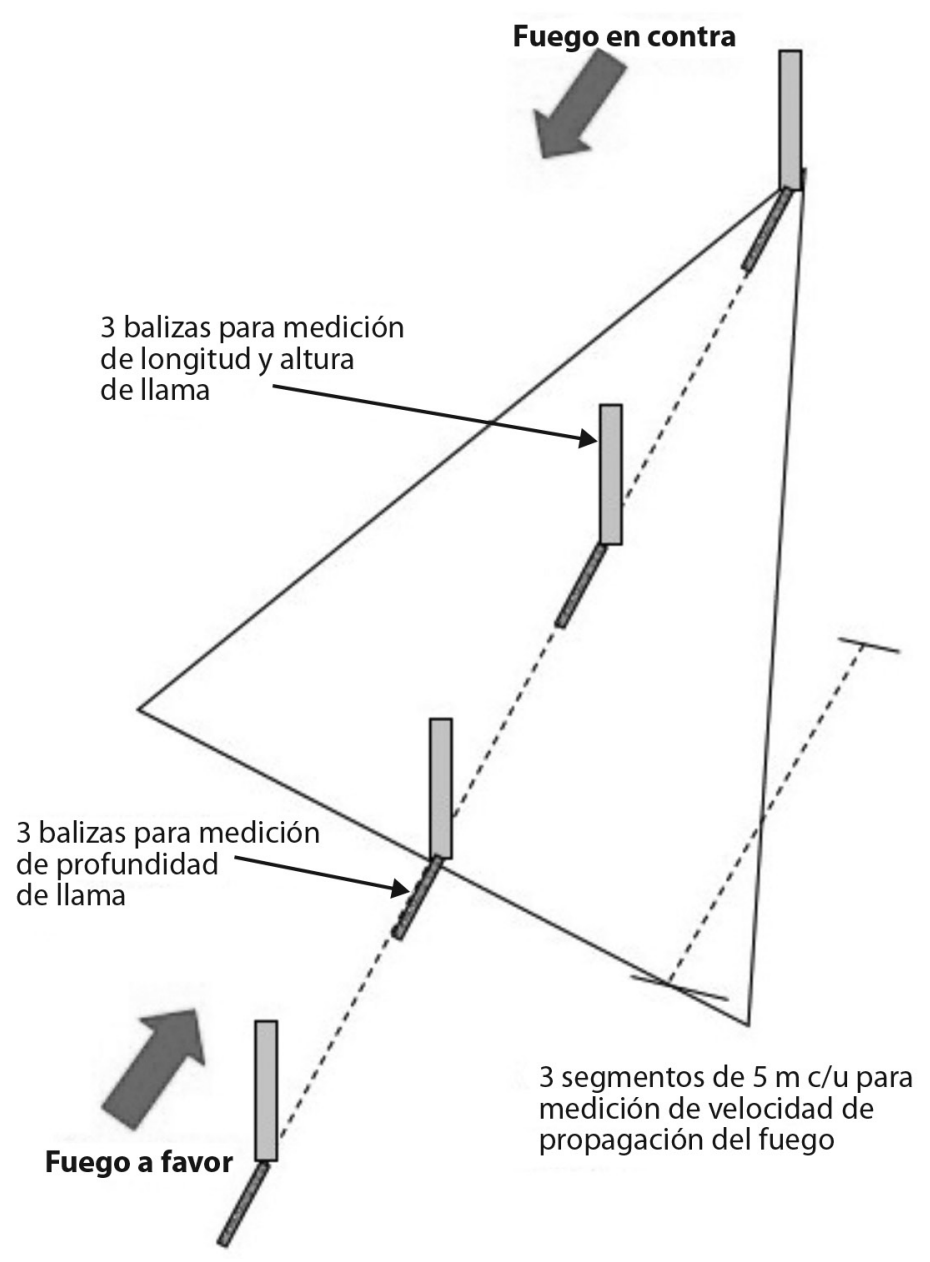

Fig. 3. Unidades muestrales para comportamiento del fuego en pastizal y sabana.

Fig. 3. Sampling units for fire behavior in grassland and savanna.

También se estimó la intensidad lineal en la línea de fuego (I), a partir de la Lll, con el modelo provisto por Alexander (1982): $\mathrm{I}=258 \mathrm{Lll}^{2.17}$.

El tiempo atmosférico fue registrado con un estuche meteorológico portátil, cuando el fuego alcanzaba cada estadal: velocidad del viento $(\mathrm{Vv})$ (anemómetro portátil) y su dirección (brújula), temperaturas (T) de bulbo seco y húmedo (psicrómetro de cadena) y a partir de éstas, con regla de cálculo, se obtuvo la humedad relativa (HR).

Estimación de emisiones de $\mathrm{CO}_{2}$ : Las emisiones de $\mathrm{CO}_{2}$ (E) fueron estimadas con el modelo de IPCC (2001): E = A C F Fe, donde: $\mathrm{A}=$ área (ha), $\mathrm{C}=$ carga de combustibles forestales ( $\mathrm{t} / \mathrm{ha}), \mathrm{F}=$ factor de consumo, y Fe $=$ factor de emisión. El Fe fue considerado igual a $1.711 \mathrm{~g} / \mathrm{kg}$, como en pastos de bosques subtropicales de Pinus palustris Mill. del SE de Estados Unidos (Aurell \& Gullett, 2015).

Diseño experimental: En el pastizal, las parcelas de quema fueron enclavadas al azar. En la sabana, se dispusieron seis parcelas de quema contiguas, tres de ellas con exposición noreste y tres con exposición suroeste. Las parcelas con diferente exposición quedaron separadas por una brecha cortafuegos sobre 
un parteaguas. Para reducir la probabilidad de escape del fuego, las tres parcelas con exposición $\mathrm{SO}$ fueron quemadas a favor de la pendiente, mientras que las restantes, con exposición NE, fueron quemadas en contra (Fig. 2).

Análisis estadístico: En todas las variables bajo estudio se detectó falta de normalidad (tendencias en gráficos Q-Q). Por esta razón se usó la prueba de rangos de Wilcoxon para comparar los tratamientos, con el procedimiento PROC NPAR1WAY del programa Statistical Analysis System (SAS ${ }^{\circledR}$, v. 9.4).

\section{RESULTADOS}

Características del arbolado: En el pastizal no hubo arbolado en las parcelas. La sabana tuvo 133.3 árboles/ha, $62.5 \%$ nances y $37.5 \%$ encinos. Los valores medios del arbolado, fueron: altura, $6.8 \mathrm{~m}(\mathrm{DE}=1.7)$, diámetro normal, $16.6 \mathrm{~cm}(\mathrm{DE}=9.2)$, diámetro a la base, $19.9 \mathrm{~cm}$ $(\mathrm{DE}=10.8)$. También se halló un área basal de $0.65 \mathrm{~m}^{2} / \mathrm{ha}$ y un área de copa igual a $410 \mathrm{~m}^{2} /$ ha $($ cobertura $=4.1 \%)$. El promedio para la altura de la cicatriz sobre el tronco fue $1.3 \mathrm{~m}$ $(\mathrm{DE}=1.5)$.

Combustibles forestales, consumo y emisiones, pastizal: La carga inicial fue $6.214 \mathrm{t} / \mathrm{ha}$, con el pasto conformando el $77 \%$ de ella. El factor de consumo total casi alcanzó la unidad y las emisiones de $\mathrm{CO}_{2}$ fueron de 10.490 t/ha en promedio (Tabla 1, Fig. 4A).

La altura y cobertura medias del pasto, fueron $0.76 \mathrm{~m}(\mathrm{DE}=0.39)$ y $0.88 \%(\mathrm{DE}=$ 0.13). El área fue homogénea $\mathrm{y}$, comparando por tipo de combustible y por carga total, solo hubo diferencias significativas para la carga inicial de la escasa hojarasca entre tipos de quema. Sus medias fueron $0.035 \mathrm{t} / \mathrm{ha}(\mathrm{DE}=$ 0.052), para parcelas quemadas a favor, y 0.135 $\mathrm{t} / \mathrm{ha}(\mathrm{DE}=0.294, \mathrm{P}=0.0122)$, en las quemadas en contra. Para la carga total, los valores fueron, respectivamente: $5.218 \mathrm{t} / \mathrm{ha}(\mathrm{DE}=1.788)$ y $7.211 \mathrm{t} / \mathrm{ha}(\mathrm{DE}=3.496, \mathrm{P}=0.2000)$.

No se reportan diferencias significativas entre las cargas de combustibles residuales, ni por tipo ni totales, entre Qc y Qf, con cargas residuales totales de $0.053 \mathrm{t} / \mathrm{ha}(\mathrm{DE}=0.046) \mathrm{y}$ $0.149(\mathrm{DE}=0.114, \mathrm{P}=0.2000)$.

Combustibles forestales, consumo y emisiones, sabana: No hubo diferencias en cargas iniciales por tipo de combustible, ni en cargas totales, al comparar entre las exposiciones $\mathrm{SO}$ (Qf) y NE (Qc), excepto en carga de arbustos. Las cargas iniciales, residuales y factores de consumo, aparecen en la Tabla 2. La carga media alcanzó 14.119 t/ha, con los zacates abarcando la mayor proporción (Tabla 2, Fig. 4B). Entre los tipos de combustible, la hojarasca mostró diferencias en la carga residual al ser quemada en contra $(0.045 \mathrm{t} / \mathrm{ha}, \mathrm{DE}=0.143) \mathrm{y}$ a favor $(0.002 \mathrm{t} / \mathrm{ha}, \mathrm{DE}=0.001)$, no así el resto
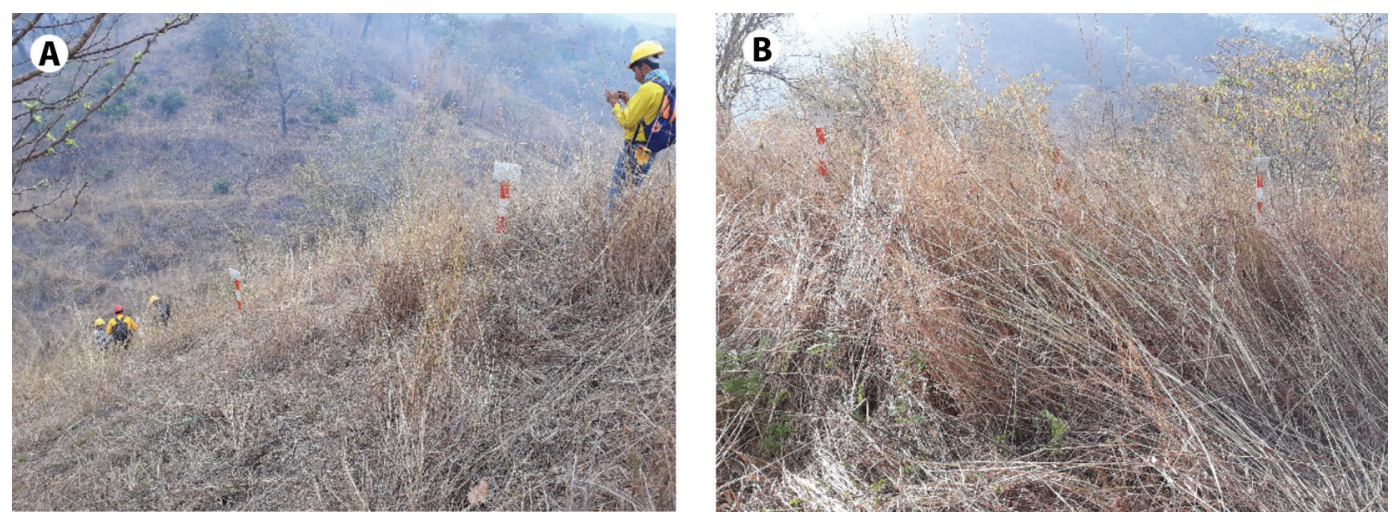

Fig. 4. Combustibles: A) pastizal (estadales de $1 \mathrm{~m}$ ) y B) sabana (estadales de $2 \mathrm{~m}$ ).

Fig. 4. Forest fuels: A) grassland (ruler of $1 \mathrm{~m}$ length) and B) savanna (ruler of $2 \mathrm{~m}$ length). 
TABLA 1

Cargas promedio iniciales, residuales, consumo y emisión de $\mathrm{CO}_{2}$ en el pastizal

TABLE 1

Initial and residual average fuel loads, consumption and $\mathrm{CO}_{2}$ emission in the grassland

\begin{tabular}{lcccc}
\multicolumn{1}{c}{ Combustibles } & Inicial $(\mathrm{t} / \mathrm{ha}, \pm \mathrm{DE})$ & Residual $(\mathrm{t} / \mathrm{ha}, \pm \mathrm{DE})$ & $\mathrm{F}$ & $\mathrm{E}(\mathrm{CO})(\mathrm{t} / \mathrm{ha})$ \\
Hojarasca & $0.085 \pm 0.116$ & $0.004 \pm 0.006$ & 0.953 & 0.139 \\
Pastos & $4.803 \pm 1.563$ & $0.087 \pm 0.105$ & 0.982 & 8.069 \\
Herbáceas dicotiledóneas & $0.007 \pm 0.016$ & $0 \pm 0$ & 1.000 & 0.012 \\
Arbustivas & $0.230 \pm 0.457$ & $0.013 \pm 0.030$ & 0.943 & 0.371 \\
Leñosos & $1.089 \pm 1.885$ & $0 \pm 0$ & 1.000 & 1.863 \\
$\quad 1 \mathrm{~h}$ & $0.032 \pm 0.078$ & $0 \pm 0$ & 1.000 & 0.055 \\
$\quad 10 \mathrm{~h}$ & $0.822 \pm 1.846$ & $0 \pm 0$ & 1.000 & 1.406 \\
$\quad 100 \mathrm{~h}$ & $0.236 \pm 0.577$ & $0 \pm 0$ & 1.000 & 0.404 \\
Total & $6.214 \pm 3.496$ & $0.107 \pm 0.103$ & 0.983 & 10.449 \\
\hline
\end{tabular}

$\mathrm{DE}=$ desviación estándar, $\mathrm{F}=$ factor de consumo, $\mathrm{E}=$ emisión.

Nota: No hubo capa de fermentación ni renuevo. 1, 10 y 100 h son los materiales leñosos por tiempo de retardo, con diámetros $<0.6$, de 0.6-2.5 y de 2.6-7.5 cm, respectivamente.

$\mathrm{DE}=$ standard deviation, $\mathrm{F}=$ consumption factor, $\mathrm{E}=$ emission.

Note: There was no fermentation layer nor tree regeneration. 1,10 and $100 \mathrm{~h}$ are the woody fuels by time lag, with diameters $<0.6,0.6-2.5$ and $2.6-7.5 \mathrm{~cm}$, respectively.

TABLA 2

Cargas promedio, residuales, consumo y emisión de $\mathrm{CO}_{2}$, en la sabana

TABLE 2

Average fuel loads, residuals, consumption and $\mathrm{CO}_{2}$ emission, in the savanna

\begin{tabular}{lcccc}
\multicolumn{1}{c}{ Combustible } & Inicial $(\mathrm{t} / \mathrm{ha}, \pm \mathrm{DE})$ & Residual $(\mathrm{t} / \mathrm{ha}, \pm \mathrm{DE})$ & $\mathrm{F}$ & $\mathrm{E}\left(\mathrm{CO}_{2}\right)(\mathrm{t} / \mathrm{ha})$ \\
Hojarasca & $2.291 \pm 2.557$ & $0.025 \pm 0.058$ & 0.989 & 3.877 \\
Pastos & $8.643 \pm 3.356$ & $0.668 \pm 1.537$ & 0.923 & 13.645 \\
Herbáceas dicotiledóneas & $0.078 \pm 0.077$ & $0.001 \pm 0.001$ & 0.987 & 0.132 \\
Arbustivas & $1.190 \pm 1.557$ & $1.179 \pm 1.540$ & 0.009 & 0.019 \\
Leñosos & $1.917 \pm 2.258$ & $0.288 \pm 0.239$ & 0.850 & 2.787 \\
$\quad 1 \mathrm{~h}$ & $0.176 \pm 0.242$ & $0.004 \pm 0.010$ & 0.977 & 0.294 \\
$\quad 10 \mathrm{~h}$ & $1.366 \pm 1.400$ & $0.284 \pm 0.235$ & 0.792 & 1.851 \\
$\quad 100 \mathrm{~h}$ & $0.376 \pm 0.920$ & $0 \pm 0$ & 1.000 & 0.643 \\
Total & $14.119 \pm 2.927$ & $2.161 \pm 2.401$ & 0.847 & 20.460 \\
\hline
\end{tabular}

Nota: En el muestreo no hubo capa de fermentación ni renuevo.

Note: In the sampling there was no fermentation layer nor tree regeneration.

de combustibles ni la carga residual total. Las emisiones de $\mathrm{CO}_{2}$ igualaron 20.460 t/ha.

Tiempo atmosférico y comportamiento del fuego, pastizal: Durante las Qf, hubo Vv de hasta $14 \mathrm{~km} / \mathrm{h}$, media de $7.7 \mathrm{~km} / \mathrm{h}$; T entre 23.3-25.6 ${ }^{\circ} \mathrm{C}$ (media, $24.3{ }^{\circ} \mathrm{C}$ ) y HR de 64 a $66 \%$, (media, $64.7 \%$ ). En las parcelas de
Qf, las pendientes variaron de $48-70 \%$, con promedio de $62 \%$.

Durante las Qc, la Vv osciló de 0 a $8 \mathrm{~km} / \mathrm{h}$ (media, $2.8 \mathrm{~km} / \mathrm{h}$ ), las $\mathrm{T}$ fluctuaron de $21.1-28.3{ }^{\circ} \mathrm{C}$ (media, $23.1{ }^{\circ} \mathrm{C}$ ) y las HR variaron entre $54-82 \%$ (promedio, $66.8 \%$ ). Las pendientes fueron de hasta $45 \%$, con un promedio de $26 \%$. 
TABLA 3

Medias y prueba de Wilcoxon de variables de comportamiento del fuego

TABLE 3

Averages and Wilcoxon test for fire behavior variables

\begin{tabular}{lcccccc}
\multicolumn{3}{c}{ Variable } & \multicolumn{3}{c}{ Pastizal } & \multicolumn{3}{c}{ Sabana } \\
& Quema a favor & Quema en contra & P & Quema a favor & Quema en contra & P \\
L11 $(\mathrm{m})$ & $3.92 \pm 1.15$ & $1.07 \pm 0.47$ & $<0.0001$ & $5.89 \pm 1.56$ & $2.21 \pm 1.22$ & $<0.0001$ \\
Pll $(\mathrm{m})$ & $1.83 \pm 0.43$ & $0.23 \pm 0.08$ & $<0.0001$ & $1.53 \pm 0.61$ & $0.76 \pm 0.51$ & 0.0126 \\
Vp $(\mathrm{m} / \mathrm{min})$ & $22.30 \pm 19.37$ & $0.67 \pm 0.26$ & $<0.0001$ & $45.5 \pm 48.1$ & $2.8 \pm 2.9$ & $<0.0001$ \\
\hline
\end{tabular}

Lll=longitud de llama, Pll=profundidad de llama y $\mathrm{Vp}=$ velocidad de propagación.

$\mathrm{Lll}=$ flame length, $\mathrm{Pll}=$ flame depth, and $\mathrm{Vp}=$ propagation rate.

Todas las variables de comportamiento del fuego mostraron diferencias significativas, resultaron mayores en las Qf (Tabla 3, Fig. 5A y Fig. 5B). En las Qf, los valores máximos fueron: $6 \mathrm{~m}$ de Lll, $2.5 \mathrm{~m}$ de Pll y una $\mathrm{Vp}$ igual a $50 \mathrm{~m} / \mathrm{min}$ en dos observaciones, estas últimas con $12 \mathrm{~km} / \mathrm{h}$ de $\mathrm{Vv}, 26{ }^{\circ} \mathrm{C}$ de T y HR de $64 \%$, así como una pendiente de $67 \%$.

Sólo una variable de comportamiento del fuego y otra de tiempo atmosférico se
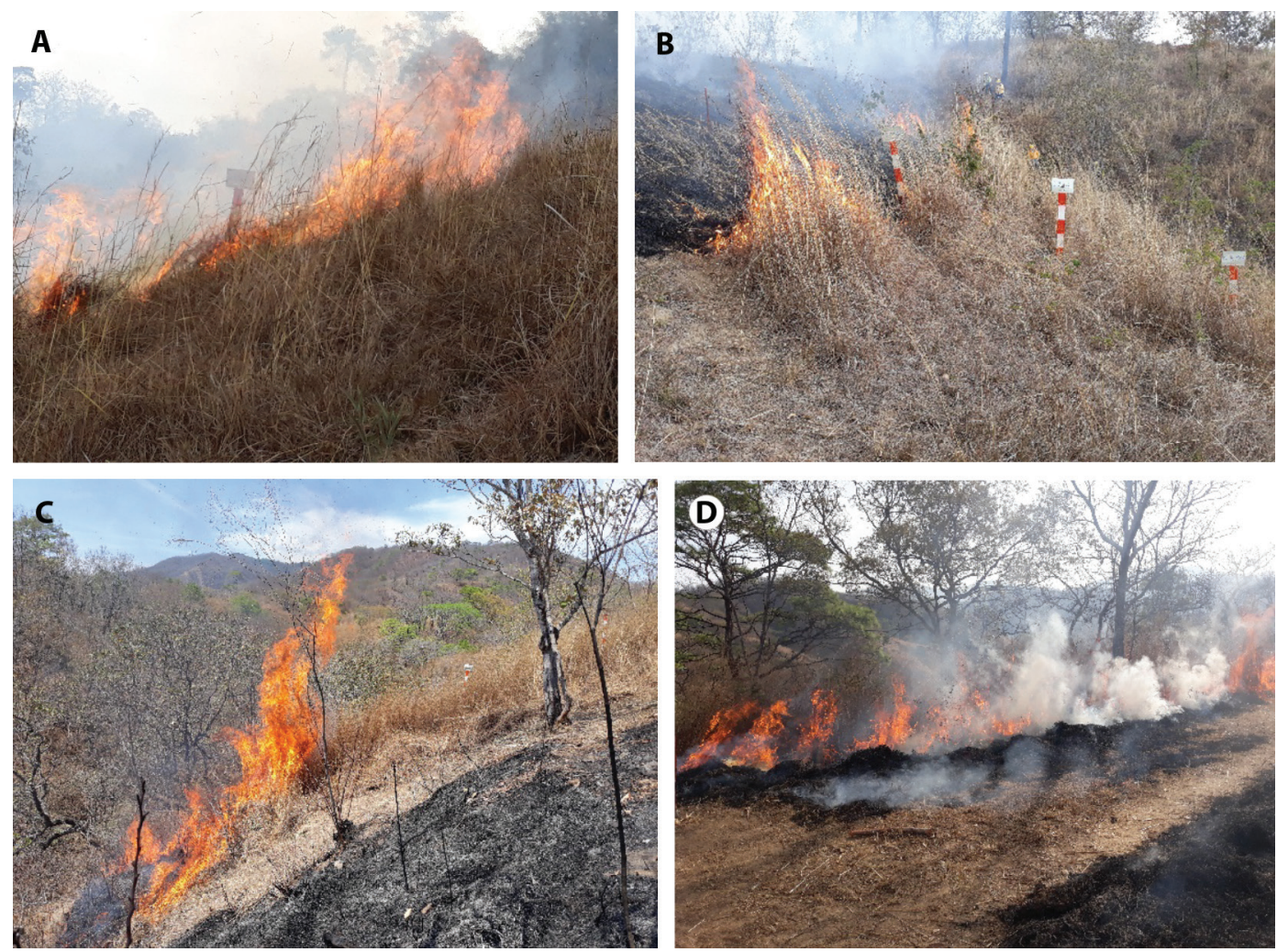

Fig. 5. Ejemplos de longitud de llama en el pastizal, A) a favor, B) en contra (estadales de $1 \mathrm{~m}$ ) y la sabana artificiales, C) a favor de la pendiente y D) en contra (estadales de $2 \mathrm{~m}$ ).

Fig. 5. Examples of flame length in the grassland, A) heading fire, B) backing fire (ruler of $1 \mathrm{~m}$ length), and in the savanna, $\mathbf{C}$ ) heading fire and $\mathbf{D})$ backing fire (ruler of $2 \mathrm{~m}$ length). 


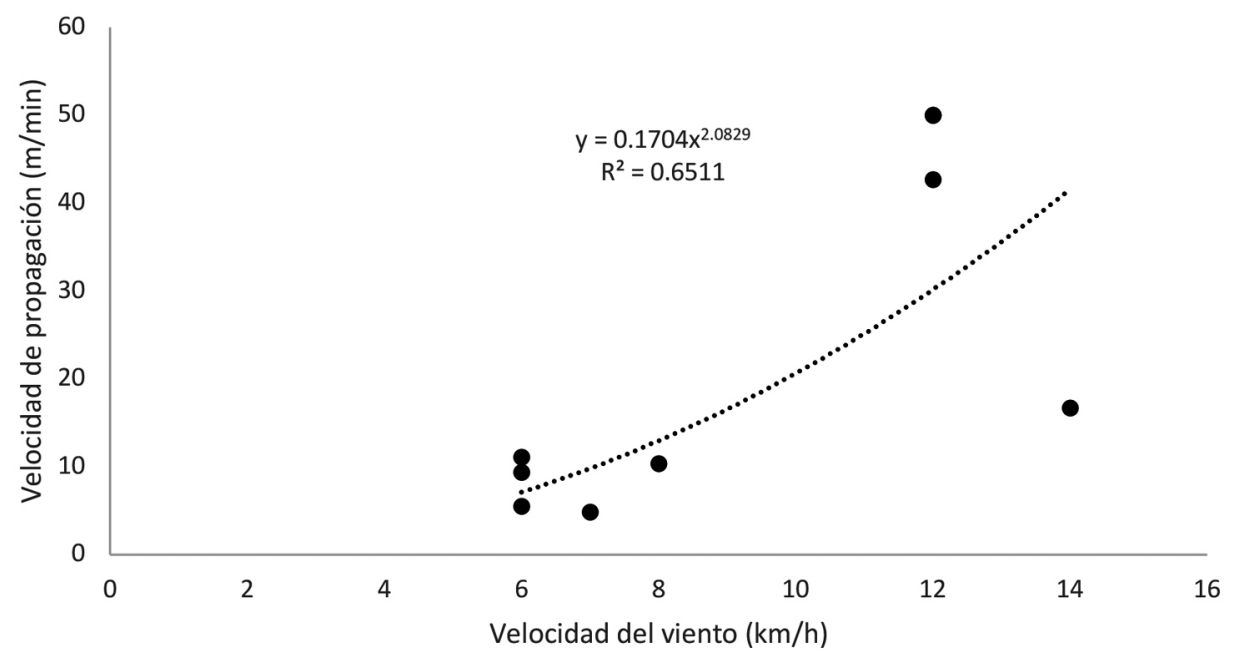

Fig. 6. Relación exponencial entre velocidad de propagación del fuego a favor y la velocidad del viento, en el pastizal. Fig. 6. Exponential relationship among heading fire rate of spread and wind speed, for the grassland.

relacionaron, la $\mathrm{Vp}$ a favor y la $\mathrm{Vv}$ en el pastizal (Fig. 6).

Tiempo atmosférico y comportamiento del fuego, sabana: Durante las quemas prescritas a favor, el viento alcanzó velocidades de 0 a $12 \mathrm{~km} / \mathrm{h}$ (media, $3.9 \mathrm{~km} / \mathrm{h}$ ); las T oscilaron de $23.9-33.9{ }^{\circ} \mathrm{C}$ (media, $30.3{ }^{\circ} \mathrm{C}$ ), y las humedades relativas variaron de 32-64\% (promedio, $45 \%$ ). Las parcelas de las Qf tuvieron pendientes entre 21 y $54 \%$ (media, $42 \%$ ).

Durante las Qc, la Vv varió de 0 a $15 \mathrm{~km} / \mathrm{h}$ (promedio, $5.1 \mathrm{~km} / \mathrm{h}$ ); las $\mathrm{T}$ de $23.3-33.3{ }^{\circ} \mathrm{C}$ (promedio, $27.9^{\circ} \mathrm{C}$ ) y las humedades relativas de 34 a $70 \%$ (media, $53 \%$ ). Aquí las laderas de las parcelas tuvieron pendientes de 47-70 \% (promedio, $59 \%$ ).

Todas las variables de comportamiento del fuego fueron diferentes entre las Qf y las Qc (Tabla 3, Fig. 6C y Fig. 6D). Los máximos observados en las Qf, fueron: $8 \mathrm{~m}$ de Lll, 2.5 $\mathrm{m}$ de Pll y $150 \mathrm{~m} / \mathrm{min}$ en $\mathrm{Vp}$; esta última con Vv de $12 \mathrm{~km} / \mathrm{h}, \mathrm{T}$ de $32{ }^{\circ} \mathrm{C}$, HR de $38 \%$ y pendiente igual a $54 \%$. La intensidad lineal del fuego para las Qf y en contra, alcanzó $12099.6 \mathrm{~kW} / \mathrm{m}$ y $1442 \mathrm{~kW} / \mathrm{m}$, con un máximo de $23513.9 \mathrm{~kW} / \mathrm{m}$.

\section{DISCUSIÓN}

En el pastizal, el pasto alcanzó una altura media de $0.76 \mathrm{~m}(\mathrm{DE}=0.39)$, la carga fue inferior a la de pastizales venezolanos de jaragua, donde a un año de aplicar una quema controlada, hubo 8.869 t/ha (Bilbao \& Medina, 1996). Tal carga también fue menor a la de pastizales amacollados (Muhlenbergia) en zonas templadas de México central, que alcanzan 7.98 t/ha (Rodríguez-Trejo \& Sierra-Pineda, 1995). Pero la carga del pastizal se ve más que duplicada cuando la especie alcanza $1.6 \mathrm{~m}(\mathrm{DE}=0.49)$ de altura (altura media máxima por sitio $=2.33$ $\mathrm{m}$, altura máxima individual $=3.20 \mathrm{~m}$ ), como en la sabana estudiada.

La menor compactación de tallos y hojas del jaragua en el pastizal y su carga relativamente ligera, son factores que favorecen la rápida de propagación del fuego. En la sabana, con 14.119 t/ha, se mantiene la baja compactación, que aunada a la mayor altura del combustible, propicia grandes Lll y veloz propagación.

Para bosques con pastizales del sur de Estados Unidos, Wade y Lunsford (1989) recomiendan llevar a cabo las quemas prescritas con una HR de 30-55\%, bajo condiciones 
especiales entre $20-60 \%$. Estos autores señalan que con una $\mathrm{HR}<30 \%$ las quemas prescritas son peligrosas, ya que el fuego es más intenso y las pavesas más probables. En contraste, con una $\mathrm{HR}>60 \%$ la quema dejará islas sin afectar y no será lo suficientemente intensa para alcanzar el nivel de consumo deseado en los combustibles y por ello tampoco se alcanzarán los objetivos de quema planteados. En nuestro caso la HR siempre fue mayor al mínimo recomendado por tales autores. Sin embargo, rebasamos el máximo, pues un frente frío se aproximó el día de las quemas de pastizal y trajo más humedad. A pesar de ello, las Qf alcanzaron intensidad importante y generaron un elevado consumo de combustibles. La velocidad promedio del viento durante las quemas fue de $1.5-5 \mathrm{~km} / \mathrm{h}$, valores recomendados por Wade y Lunsford (1989), aunque en algunos momentos se tuvieron ráfagas $>10 \mathrm{~km} / \mathrm{h}$ en las Qf. Para la realización de estas quemas prescritas de investigación, se tomaron muchas precauciones: preventivas, de recursos humanos y materiales y de comunicación, aviso y apoyo disponible en caso de escape del fuego, este último no se tuvo gracias a las previsiones tomadas.

La Lll en pastizales pocas veces alcanza más de 4 m (Scott et al., 2014), sin embargo, se trata de pastos más bajos que el jaragua. En nuestro trabajo la media se mantuvo bajo tal cota, no así el máximo de longitud de llama.

La I alcanzó 5000.9 y $298.8 \mathrm{~kW} / \mathrm{m}$, para Qf y Qc. Chandler, Cheney, Thomas, Trabaud y Williams (1983), refieren 400-425 kW/m como límite para control directo con brigadas terrestres, por lo cual un incendio forestal con las características de la quema en contra, podría ser controlado mediante combate directo (con herramientas manuales a pie de llama), pero no otro con una Lll e intensidad como las de las Qf. Para el pico de Lll observado, 6 m, la I de $12595.3 \mathrm{~kW} / \mathrm{m}$ equivale a la intensidad sostenida de incendios de copa, establecida en más de $10000 \mathrm{~kW} / \mathrm{m}$ por Scott et al. (2014).

Los incendios en pastizales son muy rápidos, especialmente en laderas pronunciadas, aún con vientos moderadamente veloces. En un incendio en Colorado hacia 1994, sobre pendientes $>55 \%$ el fuego rebasó la velocidad de $360 \mathrm{~m} / \mathrm{min}$ (Butler et al., 1998). Esta última fue más de siete veces la máxima registrada en el presente trabajo, durante Qf; aunque la pendiente en nuestro estudio también fue pronunciada, pero los vientos no soplaban veloces y la humedad relativa no era baja (64 \% como mínimo); aún así, la Vp máxima obtenida en el presente trabajo, es considerada extrema.

Los parámetros medidos son mayores en las Qf que en las Qc debido a la pendiente $\mathrm{y}$ al viento, principalmente. La pronunciada pendiente favorece que la columna convectiva seque los combustibles cuesta arriba y su rápida ignición. El viento tiende ambas, la flama y la columna convectiva sobre el frente del fuego y así seca o pone la llama en contacto directo con los combustibles que están adelante; además transporta pavesas volantes o masas de gas ardiendo, y todo esto acelera la combustión.

Los pastos son combustibles finos, con elevada relación superficie/volumen que permite una mayor superficie para el acceso de calor por cada unidad de volumen de combustible, y se facilita que alcancen rápidamente la $\mathrm{T}$ de ignición (Scott et al., 2014).

Las condiciones de tiempo atmosférico durante las quemas experimentales en la sabana artificial, también estuvieron dentro de los parámetros de HR y de Vv recomendados por Wade y Lunsford (1989), si bien hubo ráfagas con velocidad superior, pero durante las Qc.

Como en la sabana los pastos tuvieron una altura de más del doble que en el pastizal, tal altura y la mayor carga de combustibles, aunque siempre ligeros, contribuyeron a incrementar los efectos de pendiente y viento mencionados. Pastos altos, con más biomasa fina, producen llama más larga al arder en condiciones equivalentes a las de pastos bajos.

En esta sabana artificial con pasto alto, la Lll promedio a favor fue mayor a los $4 \mathrm{~m}$ dados por Scott et al. (2014) como el límite observado en pastizales, seguramente más bajos que el jaragua.

Incluso la I media de las Qc es muy superior al límite de $425 \mathrm{~kW} / \mathrm{m}$ que permite control directo, dado por Chandler et al. (1983). 
Aunque aquí se condujo al fuego en condiciones de seguridad previstas, un incendio de características similares sería simplemente imparable y peligroso para brigadas terrestres sin apoyo de equipo como carros bomba (donde hubiera acceso con seguridad para tal equipo) o apoyo aéreo.

Aunque la pendiente juega un papel toral en la Vp del fuego, por el secado de los combustibles en la parte superior de la ladera, el viento también tiene una influencia importante. En el presente trabajo, las Qf en sabana avanzaron en promedio 16 veces más rápido que las Qc, sin tratarse de vientos extremos; no obstante, tienden llama y columna convectiva, alimentan la combustión con oxígeno y generan más pavesas tanto más intenso es el fuego.

Debido a las fuertes pendientes en las áreas de estudio, y región, se recomienda hacer las quemas controladas y las prescritas en contra, con $\mathrm{T}$ de hasta $28{ }^{\circ} \mathrm{C}$ y HR de $50 \%$ o más, entre noviembre y marzo. Para pastos más altos, como los de la sabana estudiada, quemas tempranas (de noviembre a febrero), con $\mathrm{T}$ de hasta $28{ }^{\circ} \mathrm{C}$ y HR $>50 \%$ para Qc, parecen promisorias.

Con base en el comportamiento del fuego observado, solamente el fuego de pastizal en contra podría ser combatido directamente, por brigadas terrestres, pero con riesgo de generación de pavesas que podría atrapar al combatiente entre dos fuegos. Se recomienda combatir del lado ya quemado. A causa de las elevadas Lll y Vp, tanto en pastizal como en sabana, particularmente en la segunda, el combate indirecto con brecha cortafuego sería más eficiente y seguro una vez que el fuego alcance la selva baja o el bosque de pinoencino y mejor si esto se aplica del otro lado del parteaguas, cuando el frente avance en contra de la pendiente.

Por los resultados de este trabajo, se debe tomar particular atención a las siguientes situaciones de peligro para el combatiente, derivadas de las 18 dadas por el Servicio Forestal de Estados Unidos, en particular cuando se trabaje entre pastos altos como los de la sabana:
Que no participen en el combate brigadas donde ninguno de los combatientes ni el jefe de la brigada tengan experiencia de combate $u$ observaciones de comportamiento del fuego en este tipo de combustibles y topografía.

No abrir línea de control directo o indirecto, sobre la misma ladera cuando el fuego avanza a favor.

No intentar combate directo cuando el fuego avanza a favor, ni en contra en el caso de sabana; ni en contra en el pastizal si el viento sopla veloz contra la pendiente, pues la Lll y la producción de humo serán importantes.

Durante el traslado o el combate, la altura del pasto jaragua en la sabana puede obstaculizar la visibilidad, facilitándose perder de vista temporalmente el fuego y fuerzas adjuntas; los vientos y las pronunciadas pendientes facilitan la producción de pavesas rodantes y volantes que pueden dejar entre dos fuegos al combatiente, en condiciones de difícil desplazamiento.

No debe obviarse que cerca de los parteaguas se generan turbulencias de viento que complican el comportamiento del fuego. Bajo las condiciones estudiadas, el comportamiento del fuego se considera extremo, en especial en la sabana, pero lo puede ser todavía más, pues pastizales y sabanas también se asientan sobre laderas más pronunciadas, las $\mathrm{T}$ pueden ser mucho más elevadas, la HR mucho más baja, y el viento puede soplar mucho más veloz, lo que permitiría alcanzar Vp y Lll mayores a los picos de $150 \mathrm{~m} / \mathrm{min}$ y $8 \mathrm{~m}$ observados durante las quemas experimentales.

El área de estudio es tropical, lo que deja ver la necesidad de contar con parámetros para la ejecución de quemas prescritas más aproximados a este tipo de ecosistemas. El uso tradicional del fuego en quemas controladas también debe tomar en cuenta las recomendaciones, de lo contrario el peligro de incendio es elevado y hay riesgo para quienes las realizan.

Finalmente, la información generada, una vez socializada, sería de utilidad en cursos de capacitación sobre manejo del fuego, ecología del fuego, combate, comportamiento del fuego y quemas prescritas para diferentes públicos, incluidas las comunidades rurales. 
Declaración de ética: los autores declaran que todos están de acuerdo con esta publicación y que han hecho aportes que justifican su autoría; que no hay conflicto de interés de ningún tipo; y que han cumplido con todos los requisitos y procedimientos éticos y legales pertinentes. Todas las fuentes de financiamiento se detallan plena y claramente en la sección de agradecimientos. El respectivo documento legal firmado se encuentra en los archivos de la revista.

\section{AGRADECIMIENTOS}

Agradecemos a: comunidades de California y Flores Magón, sus brigadas comunitarias de prevención y combate de incendios y de medición, Ayuntamiento de Villaflores, Biomasa, A. C., Universidad Autónoma Chapingo, Universidad Autónoma de Chiapas, Universidad de Ciencias y Artes de Chiapas, FMCN, USDA FS, US AID, Conafor, Rebise, Conanp y Gobierno del Estado de Chiapas. A Mirángel Vázquez Gómez y a René Pinto Gómez.

\section{RESUMEN}

Introducción: Se utilizan quemas como parte del manejo de pastos introducidos en Chiapas, lo cual puede derivar en incendios forestales. Objetivos: obtener la carga de combustibles, comportamiento del fuego, emisiones de $\mathrm{CO}_{2}$ y derivar aspectos de seguridad durante el combate de incendios en pastizales y sabanas artificiales de pasto jaragua (Hyparrhenia rufa Nees.), debido a la falta de información sobre estos temas. Métodos: Se estudiaron un pastizal y una sabana, ambos de pasto jaragua, en las comunidades California y Flores Magón, respectivamente, en la Reserva de la Biosfera La Sepultura. Se midieron cargas de combustibles previos y posteriores a las quemas. Se condujeron seis quemas prescritas (tres a favor de viento y pendiente y tres en contra), en cada uno de los dos tipos de vegetación analizados, y se midieron variables meteorológicas, así como variables de comportamiento del fuego. Las emisiones se estimaron multiplicando la carga de combustibles consumida por una constante de emisión. Resultados: En el pastizal las medias fueron: carga inicial, 6.214 t/ha; carga residual, 0.107 t/ha, emisión de $\mathrm{CO}_{2}, 10.449$ t/ha. Para la sabana, los valores alcanzaron: 14.119, 2.161 y $20.460 \mathrm{t} / \mathrm{ha}$, respectivamente, sin diferencias para las cargas previas y posteriores a la quema entre las quemas. Para el pastizal, las quemas a favor alcanzaron: $3.92 \mathrm{~m}$, $1.83 \mathrm{~m}$ y $22.3 \mathrm{~m} / \mathrm{min}$ para longitud y profundidad de llama y velocidad de propagación, mientras que tales valores para las quemas en contra, fueron: $1.07 \mathrm{~m}, 0.23 \mathrm{~m}$ y $0.67 \mathrm{~m} /$ min, con diferencias entre tipos de quema. Para la sabana, las quemas a favor alcanzaron $5.89 \mathrm{~m}$ (longitud de llama), $1.53 \mathrm{~m}$ (profundidad de llama) y $45.5 \mathrm{~m} / \mathrm{min}$ (velocidad de propagación), mientras que para las quemas en contra tales valores fueron: $2.21 \mathrm{~m}, 0.76 \mathrm{~m}$ y $2.8 \mathrm{~m} / \mathrm{min}$, respectivamente. En todos los casos se reportan diferencias significativas. Conclusiones: El comportamiento del fuego, en particular en la sabana estudiada, es peligroso, por lo cual una buena prescripción y mucha precaución deben preverse durante quemas controladas o prescritas. Asimismo, un combate directo por el frente del fuego puede resultar muy peligroso, por lo cual debe evitarse y mejor combatir por la cola del incendio y esperar a que el frente avance en contra del viento y pendiente para combatirlo.

Palabras clave: carga de combustibles, Chiapas, $\mathrm{CO}_{2}$, comportamiento del fuego, consumo de combustibles, Hyparrhenia rufa, pasto Jaragua.

\section{REFERENCIAS}

Aguirre-Muñoz, A., Mendoza-Alfaro, R., Arredondo-Ponce-Bernal, H., Arriaga-Cabrera, L., Campos-González, E., Contreras-Balderas, S., ... Elías-Gutiérrez, M. (2009). Especies exóticas invasoras: impactos sobre las poblaciones de flora y fauna, los procesos ecológicos y la economía. En J. Sarukhán Kermes (coord. gral.). Capital Natural de México. Vol. II: Estado de conservación y tendencias de cambio (pp. 277-318). México: Conabio.

Alexander, M.E. (1982). Calculating and interpreting forest-fire intensities. Canadian Journal of Botany, 60, 349-357.

Aurell, J., B.K., \& Gullett, D.T. (2015). Emissions from southeastern grasslands and pine savannas: Comparison of aerial and ground field measurements with laboratory burns. Atmospheric Environment, 111, 170-178.

Beerling, D.J., \& Osborne, C.P. (2006). The origin of the savanna biome. Global Change Biology, 12, 2023-2031.

Bilbao, B., \& Medina, E. (1996). Types of grassland fires and Nitrogen volatilization in tropical savannas of Calabozo, Venezuela. En J.S. Levine (coord.), Biomass Burning and Global Change (Vol. 2, pp. 569574). Cambridge: The MIT Press.

Bogdan, A.V. (1997). Pastos Tropicales y Plantas de Forraje. México: AGT Editor.

Brown, J.K. (1974). Handbook for inventorying downed Woody material (Reporte Técnico General INT-16). Ogden, Utah: USDA Forest Service, Intermountain Forest Research Station. 
Butler, B.W., Barlette, R.A., Bradshaw, L.S., Cohen, J.D., Andrews, P.L., Putnam, T., \& Morgan, R.J. (1998). Fire behavior associated with the 1994 South Canyon Fire on Storm King Mountain, Colorado (Reporte RMRS-RP-9). Ogden, Utah: USDA Forest Service, Rocky Mountain Research Station.

Certini, G. (2005). Effects of fire on properties of forest soils: a review. Oecologia, 143, 1-10.

Chandler, C., Cheney, P., Thomas, P., Trabaud, L., \& Williams, D. (1983). Fire in Forestry. (Vol. 1.) Forest Fire Behavior and Effects. New York: Wiley and Sons.

IPCC (Intergovernmental Panel of Climatic Change). (2001). Climate Change 2001: Impacts, Adaptation and Vulnerability. Cambridge, U. K.: Cambridge University Press.

Lesur, L. (2010). Manual de pasturas. México: Trillas.

Leiva, J.A., Rocha, O.J., Mata, R. \& Gutiérrez-Soto, M.V. (2009). Cronología de la regeneración del bosque tropical seco en Santa Rosa, Guanacaste, Costa Rica. I. Características edáficas. Revista de Biología Tropical 57, 801-815.

Miranda, F. (2016). La Vegetación de Chiapas (Vol. 1). México: UNICACH.

Miranda, F., \& Hernández-Xolocotzi, E. (2014). Los Tipos de Vegetación de México y su Clasificación. México: Sociedad Botánica de México, Conabio, FCE.
Rocha-Loredo, A.G., Ramírez-Marcial, N., \& González Espinosa, M. (2010). Riqueza y diversidad de árboles del bosque tropical caducifolio en la depresión central de Chiapas. Boletín de la Sociedad Botánica de México, 87, 89-103.

Rodríguez-Trejo, D.A., \& Sierra-Pineda, A. (1995). Evaluación de los combustibles forestales en los bosques el sur del Distrito Federal. Ciencia Forestal en México, 20, 193-218.

Scott, A.C., Bowman, D.M.J.S., Bond, W.J., Pyne, S.J., \& Alexander, M.E. (2014). Fire on Earth. An Introduction. Singapore: Wiley Blackwell.

Secretaría de Medio Ambiente y Recursos Naturales [SEMARNAT]/Secretaría de Agricultura, Ganadería, Desarrollo Rural, Pesca y Alimentación [SAGARPA]. (2009). Norma Oficial Mexicana NOM-015-SEMARNAT/SAGARPA-2017. Diario oficial de la federación, 16 de enero de 2009, 23-91.

Villanueva Ávalos, J.F., Negrete-Ramos, L.F., VillalobosGonzález, J.C., \& Britton, C.M. (2008). Respuesta de seis gramíneas tropicales a la quema prescrita en la costa oeste de México. Técnica Pecuaria en México, 46, 397-411.

Wade, D.D., \& Lunsford, J.D. (1989). A guide for prescribed fire in southern forests. (Reporte técnico RS-TP 11). Atlanta: USDA Forest Service Southern Region. 